\title{
De un vistazo
}

Continuamos acercándoles a los lectores un breve comentario de varios artículos de publicación reciente para su lectura de acuerdo al interés individual.

\begin{abstract}
Cirugía de reparación de hernia inguinal con malla. Estudio prospectivo aleatorizado controlado de laparoscopía versus cirugía a cielo abierto. Seguimiento a cinco años.

(BMJ 2003;326:1012-1013).

La cirugía de reparación de hernia inguinal con malla por vía laparoscópica (transabdominal preperitoneal) ha demostrado ser efectiva y en comparación con la cirugía a cielo abierto menos doIorosa con un retorno más precoz a las actividades.Se realizó un estudio prospectivo aleatorizado controlado para evaluar las complicaciones a largo plazo de estas dos técnicas.El mismo se llevó a cabo en Londres, Inglaterra, entre 1995 y 1996, e incluyó a 403 pacientes.Mediante un cuestionario telefónico realizado al menos luego de 5 años de la cirugía, se evaluó la presencia de complicaciones: parestesias, dolor inguinal, dolor testicular, atrofia testicular, hernia contralateral y recurrencia. Un total de 374 pacientes estaban vivos a los 5 años, de los cuales $242(65 \%)$ fueron interrogados. El seguimiento medio fue de 5,8 años. La tasa de complicaciones fue menor en el grupo de cirugía laparoscópica (13/122) que en el grupo de cirugía a cielo abierto $(52 / 120)$. El adormecimiento de la herida $(3 / 122-3 \%$ vs. $27 / 120-23 \% ; p<0,0001)$ y el dolor inguinal $(2 / 122-2 \%$ vs. $12 / 120-10 \% ; p=0,006)$ fueron significativamente reducidos, demostrando así el estudio menores complicaciones a largo plazo con la técnica laparoscópica.Sin embargo se necesitarían grandes estudios aleatorizados para arribar a firmes conclusiones en esta materia (ver ensayo reciente de Neumayer y cols. publicado en el New England electrónico el 25 de Abril de 2004). Mientras tanto es recomendable elegir la técnica en la cual el centro cuente con mayor experiencia.
\end{abstract}

\section{Meta-análisis sobre el uso de recursos y los costos asocia- dos al uso de corticoides inhalados vs. antagonistas de leu- cotrienos en el tratamiento del asma.}

(J Fam Pract 2003;52:382-389).

El tratamiento estándar para los pacientes con asma es el corticoide inhalado. Estos han demostrado mejorar la evolución y disminuir los costos y el uso de recursos. Luego de la introducción de los antagonistas de los leucotrienos, no se han hecho estudios diseñados para comparar los efectos sobre los costos y el uso de recursos. Se seleccionaron 6 estudios para este meta-análisis, todos esponsoreados por GlaxoSmithKline. Cinco son cohortes retrospectivos y uno es prospectivo. Los pacientes que recibieron corticoides inhalados, comparados con los que recibieron antagonistas de los leucotrienos, presentaron:menor tasa de internación anual ( $2.2 \%$ vs $4.3 \%$; $p<.05$. NNT:48 por 1 año), mayor reducción de la tasa de internación luego del inicio del tratamiento $(2.4 \%$ vs $0.55 \% ; p<.01)$, menor tasa de visitas a guardia $(6.2 \%$ vs $7.7 \%$; $p<0.005)$ y menores costos relacionados al asma $(p<.05)$. Las diferencias en cuanto a costos totales no fueron significativas. Como limitaciones se mencionan que solo 6 de 49 estudios (12\%) fueron incluidos en el meta-análisis, que la mayoría de los estudios incluidos fueron retrospectivos y que son estudios esponsoreados por un laboratorio. En conclusión, teniendo en cuenta las limitaciones, el meta-análisis demuestra la superioridad en cuanto a efectividad y a costos de los corticoides inhalados sobre los antagonistas de los leucotrienos.

\section{Exposición al sol, fenotipo de piel y riesgo de esclerosis} múltiple (EM): estudio caso-control ${ }^{*}$.

(BMJ 2003;327:316-321).

La EM involucra un defecto en la autotolerancia inmunológica que conlleva el ataque a la propia mielina por los linfocitos $T$ helper tipo 1.Se observó un aumento de la prevalencia de la enfermedad con la latitud, y se la relacionó inversamente con la exposición al sol. Esto podría deberse al efecto de supresión de las respuestas de los linfocitos $T$ helper por la radiación ultravioleta y la vitamina D.Este estudio de casos y controles* realizado en Tasmania, Australia (latitud $21-3^{\circ} \mathrm{S}$ ), lugar con alta prevalencia de EM, evalúa si una alta exposición al sol en el pasado se asocia con una reducción del riesgo de tener EM. Se tomaron 136 casos y 272 controles.Se realizó un cuestionario para medir la exposición al sol y se tomaron muestras de piel como marcador objetivo de daño solar.Se determinó el fenotipo de piel por espectrofotometría. El color de piel clara fue más frecuente en los casos que en los controles, aunque en el límite de lo significativo $\left(\mathrm{OR}^{*} 1.59\right.$, IC 0.992.55). La alta exposición solar entre los 6 y 15 años y el daño actínico de la piel se asociaron a una reducción del riesgo de EM $\left(\mathrm{OR}^{\star} 0.31\right.$, IC $95 \%$ 0.16-0.59 y OR* 0.32 , IC $0.11-0.88$ respectivamente), luego de ser ajustado para otros factores relacionados a la enfermedad. Otros estudios demostraron que la deficiencia de vitamina $D$ era más frecuente en pacientes con EM. Debido a los posibles sesgos* de este tipo de estudios, sería interesante demostrar que el suplemento con vitamina $D$ reduce el riesgo de la enfermedad.

\section{Evaluación del dímero $\mathrm{D}$ en el diagnóstico de sospecha de trombosis venosa profunda (TVP)}

\section{(NEJM 2003;349:1227-1235).}

Se realizó un estudio prospectivo aleatorizado para evaluar el uso del dímero $D$ y su alto valor predictivo negativo*, como guía para la realización del doppler venoso y diagnóstico de TVP. Un total de 1096 pacientes ambulatorios con sospecha de TVP fueron categorizados clínicamente como de alta o baja probabilidad. Luego se aleatorizaron a doppler venoso o a dímero $D$. En este último grupo se realizó también doppler venoso a menos que la probabilidad clínica fuese baja y el dímero $D$ negativo, en cuyo caso se descartaba TVP.En los pacientes en los que se descartó TVP inicialmente, se diagnosticó TVP en el seguimiento a 3 meses en 0,4\%; IC 95\% 0,05 a 1,5 (grupo dímero D) vs 1,4\%;IC 95\% 0,5 a 2,9 (grupo doppler); $\mathrm{p}=0,16$ (diferencia no significativa). El uso de dímero $D$ redujo significativamente la utilización de doppler venoso, de 1,34 por paciente a 0,78 por paciente $(p=0,008)$. El $39 \%$ de los pacientes en el grupo dímero $D$ no requirió doppler venoso. Los resultados de este trabajo avalan el uso de esta estrategia con el objetivo de reducir la utilización de doppler venosos. Para la aplicación de estos resultados en nuestro medio es necesario conocer la sensibilidad y especificidad de nuestros test diagnósticos en comparación con los utilizados en este trabajo (teniendo en consideración el carácter operador-dependiente del doppler). Además se debe tener en cuenta la disponibilidad y los costos locales tanto del dímero $\mathrm{D}$ como del doppler venoso.

\section{Dr. Nicolás Cazap}

[ Residente Medicina Interna, Instituto Universitario CEMIC ]

\section{Dr. Alejandro Abbate}

[ Profesor Asistente y Coordinador de la Carrera de Especialización y Residencia de Medicina Interna - Instituto Universitario CEMIC ] 